\title{
The Number of Digraphs with Cycles of Length $k$
}

\author{
Chuanlong Wang ${ }^{1}$, Mudaster Sidik' ${ }^{2}$ Xuerong Yong ${ }^{3}$ \\ ${ }^{1}$ Department of Mathematics, Taiyuan Normal University, Taiyuan, China \\ ${ }^{2}$ Department of Mathematics, Yili Normal University, Yili, China \\ ${ }^{3}$ Department of Mathematical Sciences, University of Puerto Rico, Mayaguez, USA \\ Email: clwang218@126.net, sidik571013@163.com, xryong@dimacs.rutgers.edu
}

Received April 17, 2013; revised May 13, 2013; accepted June 5, 2013

Copyright (C) 2014 Chuanlong Wang et al. This is an open access article distributed under the Creative Commons Attribution License, which permits unrestricted use, distribution, and reproduction in any medium, provided the original work is properly cited. In accordance of the Creative Commons Attribution License all Copyrights (C) 2014 are reserved for SCIRP and the owner of the intellectual property Chuanlong Wang et al. All Copyright (C) 2014 are guarded by law and by SCIRP as a guardian.

\section{ABSTRACT}

In this note, we show that the number of digraphs with $n$ vertices and with cycles of length $k, 0 \leq k \leq n$, is equal to the number of $n \times n(0,1)$-matrices whose eigenvalues are the collection of copies of the entire $k$ th unit roots plus, possibly, 0's. In particular, 1) when $k=0$, since the digraphs reduce to be acyclic, our result reduces to the main theorem obtained recently in [1] stating that, for each $n=1,2,3, \cdots$, the number of acyclic digraphs is equal to the number of $n \times n(0,1)$-matrices whose eigenvalues are positive real numbers; and 2) when $k=n$, the digraphs are the Hamiltonian directed cycles and it, therefore, generates another well-known (and trivial) result: the eigenvalues of a Hamiltonian directed cycle with $n$ vertices are the $n$th unit roots [2].

\section{KEYWORDS}

\section{Acyclic Digraph; Eigenvalue; Power Digraph; (0,1)-Matrix}

\section{Introduction}

A digraph $G_{n}$ of $n$ vertices $\{1,2, \cdots, n\}$ is a directed graph whose edges are oriented from vertex $i$ to vertex $j$, $1 \leq i, j \leq n$. In this note, the digraphs to be considered are with loops or cycles, but parallel edges are forbidden. An acyclic digraph is a digraph that has no cycles of any length. Let $D_{n, k}$ be a digraph of $n$ vertices with cycles of length $k$ plus, possibly, an acyclic digraph with $n-m k$ vertices, where $m$ is the number of cycles, and where $k$ is fixed, $0 \leq k \leq n$. Then it is seen that the cycles in $D_{n, k}$ are disjoined (therefore all the cycles that it has are simple) and if $k<n$ the digraph is not strongly connected. And, in particular, $D_{n, n}$ is a Hamiltonian directed cycle of size $n$ and $D_{n, 0}$ is an acyclic digraph of $n$ vertices, respectively.

The acyclic directed graphs have been considered by several authors in the past decades. A first related result appeared in the literature seems to be the one described in [2]. It says that a digraph $G$ contains no cycle if and only if all eigenvalues of its adjacency matrix are 0 . Subsequently, to the best of our knowledge, Robinson [3,4] and Stanley [5] counted the acyclic digraphs independently and showed that if $R_{n}$ stands for the number of acyclic digraphs of $n$ vertices then

$$
R_{n}=\sum_{k=1}^{n}(-1)^{k+1}\left(\begin{array}{l}
n \\
k
\end{array}\right) 2^{k(n-k)} R_{n-k} \sim n ! \frac{2^{\left(\begin{array}{l}
n \\
2
\end{array}\right)}}{M p^{n}},
$$

where $p=1.488 \cdots$, and $M=0.474 \cdots$. Later, in [6,7], Bender et al. considered the asymptotic number of acyclic digraphs with $q$ edges, and subsequently, Gessel counted the acyclic digraphs by their sources and sinks in [8]. Most recently, E. Weisstein of Wolfram Research Inc. calculated the number, $M_{n}$, of $n \times n(0,1)$ matrices with real positive eigenvalues and showed that for $n=1,2,3,4,5$ the numbers $M_{n}$ are 
because the numbers were observed to coincide with the first five values of the sequence of the number of acyclic digraphs with $n$ vertices that is obtained by Sloane in [9]. Weisstein conjectured that the two sequences are identical. The conjecture has recently been proven in [1].

Motivated by the above literature, we extend the acyclic digraphs to consider the digraphs $D_{n, k}$ with cycles of length $k$ in this note, where $0 \leq k \leq n$. Our theorem established in the next section indicates that similar counting theorem holds for more general graphs.

\section{The Main Results}

Let us first prove the following lemma.

Lemma 1 Given a positive integer $n$, and a nonnegative integer $k$ with $0 \leq k \leq n$, the eigenvalues of the adjacency matrix $A=A\left(D_{n, k}\right)$ of $D_{n, k}$ are copies of the entire kth unit roots plus, possibly, 0's. Conversely, if $B$ is an $n \times n \quad(0,1)$-matrix whose eigenvalues are copies of the entire kth unit roots plus, possibly, 0 's then its digraph $D(B)$ is isomorphic to $D_{n, k}$, if ignoring the acyclic parts in the two digraphs.

Proof. Assume that $D_{n, k}$ has $m$ cycles of length $k$. We show that the eigenvalues of $A$ are $m$ copies of the entire $k$ th unit roots plus $n-m k$ 0's. Since relabeling the vertices of a graph does not change the eigenvalues of its adjacency matrix, and since the $m$ cycles of length $k$ are disjoined, we may number the vertices consistently with the partial order so that $A$ has the upper block-triangular as follows:

$$
A=\left(\begin{array}{ccccccc}
A_{1} & * & * & \cdots & * & * & * \\
0 & A_{2} & * & \cdots & * & * & * \\
& & \cdots & & & & \\
0 & 0 & \cdots & A_{m} & * & * & * \\
0 & 0 & \cdots & 0 & 0 & * & * \\
& & \cdots & & & 0 & * \\
0 & 0 & \cdots & & & 0 & 0
\end{array}\right)
$$

where each $A_{i}, i=1,2, \cdots, m$, is the adjacency matrix of a (directed simple) cycles of length $k$, and where '*' is either a block matrix of 0,1 , or 1 , or 0 's. From linear algebra, it can be easily proven that, for any $i(\leq m)$, the characteristic polynomial of $A_{i}$ is $\left|\lambda I-A_{i}\right|=\lambda^{k}-1$. So the eigenvalues of $A_{i}$ are the $k$ th unit roots. Since the eigenvalues of $A$ are collection of the eigenvalues of these $A_{i}$ and $n-m k 0$ 's, its eigenvalues are $m$ copies of the entire $k$ th unit roots plus $n-m k$ zeroes.

Conversely, if $B$ is an $n \times n \quad(0,1)$-matrix whose eigenvalues are $m$ copies of the entire $k$ th unit roots plus $n-m k$ o's, then its graph $D(B)$ is a digraph and, for any $i(\leq n)$, the ith eigenvalues of $B^{k}, \lambda_{i}\left(B^{k}\right)$, is either 1 or 0 . We now consider the power digraphs of $D(B)$ with adjacency matrix $B$. Since for all $l=1,2,3, \cdots$ ।

$$
\operatorname{trace}\left(B^{l k}\right)=\sum_{i=1}^{n} \lambda_{i}^{l k}=m k
$$

the number of closed walks of length $l$ in the $k$ th power graph $D(B)^{k}$ of $D(B)$ is $m k$. Since the eigenvalues of $B^{k}$ are either 1 or 0 , the diagonal elements of $B^{k}$ must be 1 or 0 . In fact, from Perron-Frobenius theory (e. g., [10], p. 28, (1,6) Corollary (a)), we have $B^{k}(i, i) \leq \rho\left(B^{k}\right)=1, \rho\left(B^{k}\right)$ is the largest eigenvalue of $B^{k}$, which implies $B^{k}(i, i)=1$ or 0 , and $B^{k}$ has exactly $m k$ 's on its diagonal. Thus, counting all the closed walks in the kth power graph $D(B)^{k}$ we conclude that $D(B)$ is a digraph with $m$ disjoined cycles of length $k$ plus an acyclic graph with $n-m k$ vertices. Putting the thing back to $B$ implies that $B$ is the adjacency matrix of a digraph with $m$ cycles of length $k$ plus, possibly, an acyclic digraph with $n-m k$ vertices. The proof is complete.

For $m=0,1,2, \cdots$, counting the number of the digraphs and the $(0,1)$-matrices in the above lemma, we immediately have the following

Theorem 1 For each $n=1,2,3, \cdots$, and nonnegative integer $k, 0 \leq k \leq n$, the number of digraphs $D_{n, k}$ with cycles of length $k$ is equal to the number of $n \times n \quad(0,1)$-matrices whose eigenvalues are the collection of copies of the entire kth unit roots plus, possibly, 0 's.

Note that when $k=0$, because of the one to one corresponding, this leads to an alternative proof of the above conjecture (the main theorem of [1]). That is, for each $n=1,2,3, \cdots$, the number of acyclic digraphs $D_{n, 0}$ 
is equal to the number of $n \times n \quad(0,1)$-matrices whose eigenvalues are 0 's --- from linear algebra, which is equivalent to saying that, for each $n=1,2,3, \cdots$, the number of acyclic digraphs $D_{n, 0}$ is equal to the number of $n \times n \quad(0,1)$-matrices whose $n$ eigenvalues are equal to 1 --- and from [1], which is also equivalent to saying that, for each $n=1,2,3, \cdots$, the number of acyclic digraphs $D_{n, 0}$ is equal to the number of $n \times n(0,1)$-matrices whose eigenvalues are positive real numbers.

Corollary 1 If a digraph $D$ has cycles of lengths $k_{i}, i=1,2, \cdots, t$, and the cycles are piecewise disjoined then the eigenvalues of its adjacency matrix $A$ are collection of the entire $k_{i}$ th unit roots, $i=1,2, \cdots, t$, plus 0 's. And vice versa.

\section{Funding}

This research is supported partially by The Shanxi Beiren Jihua Projects of China, DIMACS (NSF center at Rutgers, The State University of New Jersey) and The University of Puerto Rico at Mayaguez.

\section{REFERENCES}

[1] B. McKay, F. Oggier, G. Royle, N. Sloane, I. Wanless and H. Wilf, “Acyclic Digraphs and Eigenvalues of $(0,1)-M a t r i c e s, ”$ Journal of Integer Sequences, Vol. 7, 2004, \#04.3.3.

[2] D. Cvetkovic, M. Doob and H. Sachs, “Spectra of Graphs,” 3rd Edition, Johann Ambrosius Barth Verlag, Leipzig, 1995.

[3] R. Robinson, “Enumeration of Acyclic Digraphs,” In: R. C. Bose, et al., Eds., Proceedings of 2nd Chapel Hill Conference on Combinatorial Mathematics and Its Applications, Chapel Hill, Orange County, 1970, pp. 391-399.

[4] R. Robinson, “Counting Labeled acyclic Digraphs,” In: F. Harary, Ed., New Directions in the Theory of Graphs, Academic Press, New York, 1973, pp. 239-273.

[5] R. Stanley, “Acyclic Orientations of Graphs,” Discrete Mathematics, Vol. 5, No. 2, 1973, pp. 171-178. http://dx.doi.org/10.1016/0012-365X(73)90108-8

[6] E. Bender, L. Richmond, R. Robinson and N. Wormald, “The Asymptotic Number of Acyclic Digraphs (I),” Combinatorica, Vol. 6, No. 1, 1986, pp. 15-22. http://dx.doi.org/10.1007/BF02579404

[7] E. Bender and R. Robinson, “The Asymptotic Number of Acyclic Digraphs (II),” Journal of Combinatorial Theory, Series B, Vol. 44, No. 3, 1988, pp. 363-369. http://dx.doi.org/10.1137/1.9781611971262

[8] I. Gessel, “Counting the Acyclic Digraphs by Sources and Sinks,” Discrete Mathematics, Vol. 160, No. 1-3, 1996, pp. 253-258. http://dx.doi.org/10.1016/0012-365X(95)00119-H

[9] N. J. Sloane, “The On-Line Encyclopedia of Integers Sequences,” 1996-2003.

[10] A. Berman and R. J. Plemmons, "Nonnegative Matrices in the Mathematical Sciences,” 2nd Edition, Academic, New York, 1994. 